\title{
VICO, SALVATOR ROSA E LE MASCHERE DEL BAROCCO
}

\section{Le maschere e l'Unheimliche}

T 'idea barocca della maschera appare caratterizzata dalla repressione Ldelle espressioni più forti del comico e della satira sociale, e finisce per soffocare la qualità trasgressiva del riso, la sua dimensione più autentica, che, come ha scritto Baudelaire, non appare attingibile dal punto di vista della saggezza e dell'etica. ${ }^{1}$ Gli aspetti inquietanti del riso nell' ambito della cultura controriformistica vengono costretti nei limiti di un comico educativo e puramente parodistico. I comici del Seicento in ultima analisi accettano i canoni dell'etica cristiana imposti dagli ecclesiastici e dai sovrani e finiscono per sottomettersi al potere politico-religioso trasformando il teatro di strada della Commedia dell'Arte in industria del divertimento al servizio dei potenti. Questo fenomeno sarà chiarito soprattutto alla fine del Seicento, quando la Commedia dell'Arte subirà la codificazione del genere come risulta evidente nell'opera dell'abate Perrucci. ${ }^{2}$ La preoccupazione degli ecclesiastici non era rivolta tanto all'osceno e al riso in sé, quanto piuttosto alla loro destinazione. Da questo atteggiamento si svilupperà una concezione della retorica come velo e "fingimento" con finalità educative dei sensi, in primo luogo della vista, il senso principe del barocco, attraverso un ampio ricorso all'emblematica. ${ }^{3}$ In realtà alla censura delle rappresentazioni ufficiali e delle immagini pubbliche corrispose poi un atteggiamento di tolleranza nella sfera privata, dove non era raro il caso di rappresentazioni a soggetto osceno o inquietante in luoghi remoti delle case nobiliari come il Palazzo granducale mediceo. ${ }^{4}$

La maschera e il teatro barocco confermano che all'origine di ogni attività scenica, sia essa festa, spettacolo o teatro, c'è sempre un fatto di esclusione, che permette al discorso del potere di affermare la non rilevanza e al limite la non esistenza di quanto mostrato dalla scena immaginaria. Lo "spazio" della maschera è quello che Jeannine Jallat ha chiamato le déplacement, la separazione, l'intervallo, la dislocazione, il fuori e il dentro. In un primo tempo il senso della maschera si incontra nello spazio esterno, ma la consapevolezza di un "fuori" rinvia all'esistenza certa di uno spazio interno, nascosto. ${ }^{5}$ La maschera, strumento privilegiato della scena immaginaria, 
nasconde ma al tempo stesso rivela l'esistenza di un mondo Altro, estraneo e non dialettizzabile, che in termini freudiani si può definire Unheimliche. In questa ottica la maschera fa riemergere quella differenza e quella diversità che non appare articolabile in termini discorsivi e che la scena immaginaria tende di per sé a dissimulare. ${ }^{6}$

Il perturbante, o inquietante è una sorta di spaventoso che risale a ciò che ci è familiare. Unheimlich è una variante di Heimlich (segreto, nascosto) e rimanda a ciò che avrebbe dovuto rimanere nascosto e che invece affiora, viene a galla inaspettatamente. L'Unheimliche riguarda non solo la storia individuale, ma anche quella collettiva. Da un punto di vista antropologico si deve parlare del processo di rimozione di atteggiamenti psichici che erano familiari ai primi uomini. Una situazione perturbante si ha ad esempio quando il confine tra la fantasia e la realtà diventa così labile da non poterne stabilire i contomi. Si tratta, dunque, di una situazione che è comune nell'infanzia e che era costitutiva della vita psichica dei primitivi. L'analisi di Freud ha condotto a vedere fonti del perturbante sia nell'antica concezione propria dell' animismo caratterizzata dagli spiriti umani che popolavano il mondo, sia nella presenza di un aspetto "demoniaco" nella vita psichica umana, caratterizzato dalla coazione a ripetere che procede dai moti pulsionali a dispetto del principio di piacere.

Dal punto di vista del perturbante freudiano l'origine demoniaca delle maschere avanzata per esempio dal Nicolini a proposito di Arlecchino, risulta più pregnante della tesi che sottolinea invece l'origine caricaturale delle maschere come quella di Pulcinella, che, secondo il Bragaglia, nascerebbe dall'imitazione caricaturizzata dei "porri" villaneschi. ${ }^{7}$ Ė stato Paolo Toschi nel suo Le origini del teatro italiano a sottolineare con forza le origini demoniache anteriori alla Commedia dell' Arte della maschera di Pulcinella e di quelle di Arlecchino e Zanni. ${ }^{8}$ In realtà per Toschi tutte le maschere hanno un'origine demoniaca e ultramondana, come è testimoniato dalla stessa etimologia longobarda della parola masca che in origine significava "morto" e che poi è passata a significare "strega": la parola maschera fin dalle sue origini ha dunque indicato un essere infernale, strega, anima di morto o esseri simili. Questa origine infernale si spiega col fatto che il Carnevale è una festa propiziatoria della fertilità della terra: le potenze della generazione sono divinità sotterranee, diavoli, le anime degli avi. ${ }^{9}$ Le maschere subiscono poi una lenta e variegata metamorfosi "umana" nel corso 
dei secoli. Per esempio il processo di "umanizzazione" e di psicologizzazione della maschera di Pulcinella si accelera nel corso dell'Ottocento ad opera del più grande attore di teatro che l'ha interpretata: Antonio Petito. In questo modo Pulcinella diventerà addirittura un'espressione borghese dei buoni sentimenti. $^{10}$

Storicamente lo spirito demoniaco della maschera sopravivive soprattutto negli zanni, cioè nei servi astuti o stupidi che popolano la Commedia dell'Arte, esprimendosi attraverso la beffa, il gioco, il lazzo mimico e verbale, il gesto scurrile. In questo contesto la maschera di Pulcinella introduce un tipo di sensualità e di desiderio erotico che si allontanano decisamente dalle immagini petrarchesche dell'amore, anche se questo atteggiamento appare inserito nel quadro di una "dissimulazione onesta" che mira a salvaguardare un tipo di etica sociale formalistica. La confusione introdotta dalla maschera nel "gran teatro del mondo" non mira insomma a turbare l'ordine, ma a riconfermarlo, anche attraverso l'iteratività del tipo che è costitutiva della maschera e mira a confermare l'orizzonte di attesa del pubblico. ${ }^{11}$ In questa maniera, l'Altro, il diverso, il contenuto inquietante di cui la maschera è rivelatrice finisce per essere esorcizzato piuttosto che rivelato dalle maschere della Commedia dell'Arte che pure hanno favorito sia pure indirettamente la penetrazione nella cultura aristocratica di elementi carnevaleschi.

In una poesia del 1723 dal significativo titolo: "Origine, progresso e caduta della poesia", Vico riconosce il carattere vuoto e dissimulatorio delle maschere e del teatro barocco, dove l'Olimpo prende il posto del Cielo e le divinità pagane come Giunone e Venere dominano la scena che spesso riprende la forma mitologica della lotta tra gli dei. Questo testo descrive la storia della poesia dalle origini eroiche al declino umano. È lo stesso percorso descritto nella Scienza nuova, e anche qui Vico insiste sul venir meno della sostanza delle immagini create dalla fantasia, ora ridotte a pure "maschere", parole prive di alcun valore "religioso" e metaforico:

or son maniere di laudar volgari, quai maschere talor senza subbietto di Diane, di Veneri e di Alcidi .... ${ }^{12}$

Le maniere "volgari" sono una conseguenza dell'involgarimento delle "alte immortali opre d'ingegno" delle Muse, che non abitano più sul Pindo né in Parnaso, ma tra gli uomini. Nel mondo secolarizzato, scrive Vico, non 
si parla più di Clio, la musa che narrava la storia eroica cominciata con Apollo che insegue Dafne, trasformata in lauro per intercessione degli dei. Il "seguire" di Apollo nel mondo eroico era proprio di un dio e il fuggire di Dafne era proprio di una fiera. Nel mondo volgare e umano, con la corruzione dei miti, "avvenne che il seguire d'Apollo fu d'impudico, il fuggire di Dafne fu di Diana". In questo modo Vico intende polemizzare con il racconto che del mito fa Ovidio, affermando che la fuga di Dafne in seguito alla corruzione del mito fu interpretata come la fuga di una dea casta di fronte a un impudico, mentre la realtà era l'opposto. ${ }^{13}$ Le dee sono diventate "maschere senza subbietto", conclude il filosofo napoletano.

Siamo lontani dalla concezione delle maschere diffusa presso gli antichi che viene tematizzata da Vico in una lettera a Giuseppe Pasquale Cirillo e poi ripresa nella Scienza nuova. Nella lettera del Vico "intorno alle maschere degli antichi", pubblicata a suo tempo dal Croce, da una parte si accenna al fatto che la prima maschera fu quella di Satiro, e dall'altra, si ricostruisce l'etimologia della voce persona che per i primi latini significava "vestir di pelli". Vico accenna anche alle maschere degli istrioni che recitavano nelle tragedie greche e latine. Egli scrive che i satiri

in quella rozzezza e semplicità dovettero ritruovare la prima maschera col vestire i piedi, le gambe e cosce di pelli caprine, che dovevan aver alla mano e tingersi i volti e 'l petto di fecce d'uva, ed armar la fronte di corna. ${ }^{14}$

La tragedia secondo Vico sembra aver avuto origine proprio da questo coro di satiri, costituito da contadini "rozzamente mascherati" che portavano le uve sui loro carri ed erano autorizzati ad ogni sorta di villania e di trasgressione. L'etimologia vichiana trova una conferma nel fatto, ricordato dal Toschi, che anticamente la maschera di Zanni assumeva figura animalesca ed era costituita da pelli di animali. ${ }^{15}$ La recente analisi storica ha mostrato come la pratica di vestirsi di pelli e di mascherarsi da animali sia da collegare a "un correlativo rituale delle metamorfosi in animali vissute in estasi". Una parte di queste consuetudini che si localizzavano alle calende di gennaio "esprimevano nel linguaggio del rito i miti rivissuti dagli uomini e dalle donne che visitavano periodicamente, in estasi, il mondo dei morti". ${ }^{16} \mathrm{Si}$ trattava di un rito di fertilità che in qualche modo viene rievocato anche nel "vestir di pelli" di cui parla Vico, che concentra la sua attenzione proprio sul 
significato di questo mascherarsi da animali che sta all'origine della tragedia, esaltandone la componente "naturale" piuttosto che quella demoniaca. Delle tre categorie di maschere diffuse nel mondo greco, la Gorgone, il satiro e Dioniso, Vico analizza soprattutto quella del satiro, anche se non mancano i riferimenti alla Gorgone, "inchiovata allo scudo di Perseo" che viene interpretato da Vico come allegoria dell' "imperio delle leggi", che trasformava in sasso coloro che lo guardavano con le "spaventose pene". ${ }^{17}$ Vico non fa invece riferimenti diretti alla maschera di Dioniso, anche se ricorda come fosse proprio Bacco, dio della vendemmia, a comandare ad Eschilo di comporre tragedie. E noto, infatti, che la maschera è il simbolo più eloquente della frenesia del dio Dioniso, e che egli nella festa della vendemmia era presente in figura di una maschera. ${ }^{18}$ In questo senso Vico intende sottolineare il carattere necessario e naturale delle maschere degli antichi, il loro rinviare a miti autentici e a riti di fertilità, a differenza delle maschere moderne, che gli apparivano invece vuote di contenuto e prive di una precisa ragione d'essere. In questi rilievi Vico si avvicina a quanto viene oggi affermato dall'antropologia e dagli studiosi di mitologia. Károly Kerényi ha scritto, ad esempio, che la maschera di Dioniso "appartiene originariamente alla natura selvaggia" e che in origine "maschera e ambiente selvaggio sono inseparabili". ${ }^{19}$

\section{L'apologia della menzogna e l'elisione del soggetto}

La maschera moderna, che pure in un primo momento è costruita di pelle, più che ad uno spontaneo "vestirsi di pelli", o al travestimento naturale, rimanda all'idea di inganno e di menzogna. Di questo rimane testimonianza nella cultura figurativa del Seicento, per esempio nel famoso quadro di Salvator Rosa intitolato La menzogna (1649 ca.) che si trova alla Galleria Pitti a Firenze. L'uomo che campeggia al centro del quadro solleva con la mano destra una maschera, che mantiene una pronunciata somiglianza con le sue caratteristiche somatiche e fisiognomiche, chiuse in una rigida fissità inespressiva e priva di vita. Il quadro pone in maniera originale il problema della verità del soggetto, nel contesto della tendenziale "elisione del soggetto", tipica dell' affermarsi della episteme classica tra XVII e XVIII secolo. Foucault vede un'affermazione tipica di questa nuova episteme ne Las Meninas (1656) di Velásquez, in cui l'autore mostra se stesso nell'atto di guar- 
dare l'osservatore, mentre i suoi veri modelli, il re e la regina di Spagna, vengono rappresentati solo indirettamente, attraverso il riflesso di uno specchio posto sulla parte posteriore dello studio. I veri soggetti del quadro sono nascosti e in questo tipo di rappresentazione Foucault riconosce l' affermarsi di un tipo di conoscenza in cui il soggetto è tenuto da parte. ${ }^{20}$

La moltiplicazione dei punti di interesse ottico è tipica della cultura barocca e si oppone alla centralità della figura di ascendenza rinascimentale. ${ }^{21}$ Questo è evidente anche in un altro importante quadro di Velásquez, Le filatrici (Las Hillanderas, $1647 \mathrm{ca}$.) che si trova al Prado. Tra l'altro, in questo quadro è possibile riconoscere un "abbassamento" del mito, una sua volgarizzazione, come avrebbe detto Vico. Infatti il tema mitologico di Minerva e di Aracne viene lasciato in secondo piano, mentre il primo piano è occupato dalla rappresentazione di una scena di vita quotidiana, il laboratorio delle filatrici.

La volontà di porre il soggetto in secondo piano è riconoscibile anche nella Menzogna di Salvator Rosa. In questo quadro, nonostante la posizione centrale assunta dalla figura, il primo piano spetta proprio alla maschera che viene esibita apertamente in quanto tale, e finisce per assorbire al suo interno il volto, il dato naturale della fisiognomia. Vien fatto di ricordare a questo proposito la definizione di maschera data dal piacentino Pio Rossi, generale della Congregazione dei monaci Eremitani di S. Gerolamo d'Italia e contemporaneo di Salvator Rosa:

Le cose del mondo hanno diversi volti, né facile è il sapere quale sia il vero: tanto l'artifizio sa al vero imitar la natura. Il tollerato abuso del mondo mette la maschera su 'l volto, perche' col portar due faccie rimangono gli uomini senza faccia; e sfacciatamente operando, col vestirsi dell'altrui volto perdono il proprio. ${ }^{22}$

È noto che nel ritratto barocco si perde l'attenzione per il viso e per i tratti fisiognomici: tutte le espressioni dell'individualità vengono considerate di ostacolo all'espressione impersonale di una ritrattistica che privilegia sempre più l'insieme della testa, il viso tende ad essere sommerso nell'apparenza generale del costume. Il ritratto non si presenta come un'arte veritiera, ma tende a mostrare il dover essere dell'uomo, ciò che lui crede o vuole essere. L'azione teatrale e persuasiva fondata sugli addobbi e sugli apparati agisce in maniera efficace sullo spettatore che viene invitato a riconoscere 
i segni distintivi della posizione sociale cui il quadro si riferisce. Solo nella patria di Rembrandt la maschera barocca viene ad essere eliminata a favore di una ricerca sul viso che sia anche indagine di un paesaggio interiore. ${ }^{23}$ D'altra parte Salvator Rosa nei suoi ritratti rifiuta di dare vita a forme mimiche tipiche e generali, e si collega piuttosto alla fisiognomica teorizzata dal napoletano Giovan Battista della Porta, che afferma l'individualità dei lineamenti facciali. ${ }^{24}$ Per questa ragione sembra di dover sottolineare il contenuto filosofico de La menzogna che tra l'altro va messa in relazione con un altro quadro di Salvator Rosa, Il Ritratto di poeta (1650 ca.).

$\mathrm{Ne}$ La menzogna viene tematizzato il paradosso della distinzione tra maschera e faccia: il fatto che il riconoscimento delle caratteristiche fisiognomiche individuali e peculiari possa essere inibito dalla presenza della maschera. L'essenza del personaggio che sta al centro del quadro non può più essere nel volto, che rimane uno schema compositivo privo di ontologia. Infatti il confine della soggettività e dell'oggettività nel volto non è chiara e solo lo sguardo, chiuso nella sua espressione angosciata, sembra alludere ad una persistente ontologia ed essenza del personaggio, contrapponendosi in maniera marcata alla maschera. Da questo punto di vista il quadro richiama l'attenzione anche sull'arte dell'attore, di cui Salvator Rosa aveva fatto una esperienza personale nel 1639 , quando nella sua ricerca di contatto con il mondo Altro da quello intellettuale in cui viveva, aveva assunto una maschera buffonesca, quella di un supposto Pasquariello, e aveva cominciato a vagabondare per le piazze delle città italiane, dando vita assieme a un gruppo di ciarlatani a una serie di rappresentazioni teatrali improvvisate nello stile della Commedia dell'Arte. ${ }^{25}$ Rimasero famose le sue interpretazioni di Coviello, da lui descritto come astuto, intrigante, falso e millantatore, ad imitazione di alcuni lineamenti di Trasone, il famoso personaggio del teatro di Terenzio. L'abilità dell'attore consiste proprio nel farsi riconoscere come una persona diversa a seconda del ruolo che svolge, della maschera che assume. L'attore non cerca la bella apparenza e nemmeno il vero aspetto del personaggio interpretato. Si accontenta di riprodurne il carattere verosimile: per questo Nietzsche metterà in luce la parentela che lega l'attore all'oratore che fa del verosimile l'oggetto stesso del linguaggio. ${ }^{26}$ In entrambe queste figure l'idea moderna e barocca della maschera allude al carattere paradossale del senso. Le maschere dei moderni, per dirla con il Vico, testimoniano che il senso non ha più un riferimento $\mathrm{e}$ un'origine "naturale", $\mathrm{e}$ 
rappresenta una ricerca che necessariamente deve passare per il sistema dei segni.

Nella cultura barocca la maschera, come del resto la metafora, è l'oggetto preferito di quello che Richard A. Lanham ha chiamato homo rhetoricus, l'uomo che pone al centro della propria esistenza il linguaggio e lo manipola come un attore sempre pronto a cambiare travestimento. ${ }^{27} \mathrm{Ma}$, a differenza di quanto avviene nel Tesauro che valorizza all'estremo la componente ludica dell'homo rhetoricus, ne La menzogna di Salvator Rosa la moltiplicazione dei punti di interesse ottico, la divisione delle linee di luce che illuminano da una parte il volto e dall'altra la maschera, allude ad una polarizzazione dell' esistenza individuale, al suo costituirsi come progetto esistenziale che si divarica da una parte verso l'esteriorità della prestazione sociale e professionale e dall'altra verso un'interiorità che non conosce se stessa e rimane chiusa nell'enigma inquieto del volto. Si comprende bene allora come non sia un caso che il Blumenberg abbia parlato del barocco come dell'epoca dell' "uomo nascosto" dell'antropologia, che succede all'epoca del "dio nascosto" della teologia. ${ }^{28}$ La maschera nel quadro di Salvator Rosa entra così nell'alveo della scena simbolica e finisce per alludere in maniera esemplare a qualcosa di estraneo e inquietante, all'ambiguità del doppio, a ciò che viene rimosso nelle apparenze e non può essere ridotto nei termini del discorso. Infatti, come si è visto, una condizione particolarmente favorevole al sorgere di sentimenti perturbanti e inquietanti si verifica di fronte all'incertezza intellettuale se qualcosa sia o non sia vivente, reale o fantastico. ${ }^{29}$ Oltre che all' Unheimliche freudiano, si può pensare a questo riguardo al totalmente altro, al tremendum et fascinans, la nota definizione del sacro di Rudolf Otto. ${ }^{30}$

D'altro canto, come si è detto, La menzogna di Salvator Rosa viene di solito messa in relazione con il Ritratto di poeta, con cui forma un dittico. Tutto questo sembra alludere proprio al fatto che il travestimento non rimane una prerogativa dell'attore, ma si estende ad altri artisti, ad altre figure sociali. La stessa lotta politica del Seicento è caratterizzata dalla dissimulazione e dal travestimento, e le corti dei principi appariranno al Traiano Boccalini dei Ragguagli di Parnaso come "botteghe di maschere, dove non si mercanteggia se non roba finta, fabricata per servizio dell'inganno". Mentre uno dei trattati sul comportamento e il costume caratteristici dell'epoca è il Della dissimulazione onesta (1641) di Torquato Accetto, che tuttavia mantiene una 
concezione metafisica e platonica della verita e distingue tra la dissimulazione che è velo e la menzogna che è nebbia o nube. Il trattato di Accetto presenta comunque una teorizzazione della duplicità e dell' ambiguità e mostra gli aspetti inquietanti dell'anima barocca, la sua tendenza a vivere la vita e la morte come gioco allusivo di finzioni sospese nel movimento ambiguo dei contrasti e delle corrispondenze. Nel capitolo IX del trattato di Accetto la bellezza corporale viene descritta come una sorta di dissimulazione del disfacimento cui va incontro il corpo umano invecchiando. La bellezza del corpo insomma non sarebbe che una dissimulazione della morte, del cadavere che si nasconde dietro le piacevoli apparenze. La dissimulazione si presenta allora come una sorta di esercizio spirituale di un'anima che rinuncia alla "salvezza" terrena e si consegna al giudizio finale divino. L'oggetto intimo della dissimulazione si rivela dunque nell'angoscia del vivere. ${ }^{31}$ D'altro canto il legame tra poesia e politica consiste nella finzione che rappresenta l'essenza di entrambe e della vita stessa perché, come scrive Pio Rossi, "chi non sa fingere non sa vivere":

È stata la finzione dell'arte poetica introdotta, con questo che resti sempre occulta e segreta. [...] Poco o nulla s'apprezza la poesia, e pure tutt'il mondo vive di poesia: poiché in tutt'il mondo si finge. Coloro che dissero che la poesia recava danno alla politica, a giudizio de' più intendenti non l'intesero bene: perché la politica non è altro, nell'uso presente, che poesia, cioè finzione, simulazione, inganno e tradimento. $^{32}$

Non a caso si trovano analoghe formulazioni retoriche sul carattere lecito della menzogna in un poeta come Giambattista Marino e in uno studioso di retorica come Torquato Accetto:

lecita è la menzogna anco talvolta quanto giova a chi mente il dir bugia e non noce il mentire a chi l'ascolta. ${ }^{33}$

Percioché a le volte è onesta anche la bugia, quando giovando a chi la dice, non nuoce a chi l'ascolta. ${ }^{34}$

In questo clima si diffonde la convinzione che sono le menzogne, le 
falsità espresse attraverso maschere e veli di vario tipo a fondare la civiltà e i rapporti sociali. Questa idea viene espressa dal poeta Giuseppe Battista in una comunicazione letta all'Accademia napoletana degli Oziosi e pubblicata poi nel 1673 con un titolo alquanto significativo: L'Apologia della menzogna. In quest'opera si sostiene appunto che la verità produce odio $\mathrm{e}$ corrode i rapporti sociali, mentre la menzogna è "genitrice di affetto" e si trova al centro della vita sociale, in un mondo fatto di pure apparenze. La dissimulazione crea una condizione di invisibilità per gli individui onesti che assieme alla nobilitazione del silenzio come arte di prudenza si presenta come vera e propria tecnica di sopravvivenza, in un secolo che non conosce la vita libera e affida all'autorità e alla Ragione di stato le proprie certezze e le proprie scelte. ${ }^{35}$

\section{Le muse ingannatrici e le muse inquietanti}

L'atteggiamento di Vico e prima ancora quello Salvator Rosa si muovono in una direzione opposta a quella del secentismo votato alla celebrazione della menzogna. È il caso qui di ricordare che l'antisecentismo nasce contemporaneamente al secentismo, e tra i primi intellettuali a polemizzare contro i "vizi" e le "infamie" del barocco e del marinismo è proprio Salvator Rosa. ${ }^{36}$ Del resto in quegli anni a Napoli si assiste ad una vera e propria guerra al marinismo. Il Nicolini racconta come intorno al 1680 si diffondessero schiere di giovani poeti che si impegnano in una campagna contro il "secolo corrotto" tale da non escludere i mezzi violenti nella ricerca della "conversione" dei rimatori ad uno stile più sobrio. A questo scopo si usavano persino le bastonature e i duelli. Rimane famosa la clamorosa conversione di Giulio Acciani ad opera del conterraneo Lionardo di Capua. A questo clima antisecentista partecipa anche Vico, che nella sua Autobiografia racconta la propria "conversione" ai modelli classici della poesia latina e volgare dopo un periodo giovanile in cui aveva poetato nelle maniere "corrotte" del barocco, introdotte a Napoli dal leccese Giuseppe Battista (1610-1675), dal catanese Giuseppe Artale (1620-1679), e da colui che ispirò maggiormente Vico: Iacopo Lubrano (1631-1693). ${ }^{37}$

Nel Teatro della politica (1669) di Salvator Rosa la politica viene presentata come l'arte che regola la vita umana, soprattutto sul piano delle relazioni individuali e interpersonali, nell'ambito di un'alta considerazione 


\section{VICO, SALVATOR ROSA E LE MASCHERE DEL BAROCCO}

della moralità e della vita interiore. Al centro del Teatro della politica non stanno i problemi dello Stato e della scienza politica moderna, ma il biasimo per l'adulazione, il culto dell'amicizia e della sincerità, cui si unisce la condanna di ogni finzione in arte. La religiosità di Salvator Rosa è tutta interiore e fondata sulla preghiera. È significativo che alla preghiera siano dedicati alcuni degli aforismi del Teatro della politica. ${ }^{38}$ In realtà la direzione di Salvator Rosa, come del resto quella del Vico, è quella di un approfondimento filosofico e critico della problematica secentista. Questo è evidente soprattutto nella sua produzione figurativa, dove i problemi posti dalle maschere del barocco non vengono tematizzati in chiave moralistica, anche se non mancano accenni in questo senso, come in questo aforisma: "Ful inventate le maschere dalle donne per incarire le bellezze del suo , agl'amanti". ${ }^{39}$

In realtà l'inquietudine del personaggio che sta al centro de La mer gna, il suo richiamare l'attenzione alla maschera in primo piano puntanc con l'indice della mano sinistra, ha origine dalla consapevolezza ch menzogna non nasce semplicemente dalla volontà umana, come volev tradizione risalente ad Agostino, che aveva scritto: "mendacium est enun tio cum voluntate falsum enuntiandi". ${ }^{40}$ In questo senso, la cultura barc si allontana dal moralismo cristiano e comprende la possibilità di una $\mathrm{m}$ zogna non come "duplex cogitatio", ma come "duplex oratio", rimanda a considerazioni di carattere linguistico, per cui si dà menzogna qua dietro alla frase menzognera che viene espressa si nasconde una frase che invece non viene espressa e che si oppone alla prima. ${ }^{41}$ La verita for mentale del barocco è una nuova concezione della vita, che si sente ser più come una tensione costante tra due poli solidali e al tempo stesso opposti. Tutto questo induce a un certo relativismo che si esprime nella dicotomia barocca che todo es verdad y todo mentira. In fondo, La menzogna di Salvator Rosa rappresenta una versione di questa dicotomia: il personaggio diventa maschera e viso, dando vita ad una serie di problemi di identità che trova espressione privilegiata nella Commedia dell'Arte e nella comedia barocca, dove la confusione dei nomi, gli inganni, i travestimenti e gli scambi di identità sono all'ordine del giomo, come accade nel famoso esempio costituito da El alcaide de si mismo, di Calderón. ${ }^{42}$

La "frase" che si nasconde nel quadro di Salvator Rosa, a dispetto della spontaneità con cui viene esibita la maschera, non consiste solo nell'enigma 
del volto, ma anche nella mancanza di un qualunque riferimento alla realtà originaria della poesia sublime e delle passioni violente e degli antichi istinti che caratterizzavano l'esistenza umana, che Salvator Rosa cercherà invece di esprimere in quadri come Streghe e incantesimi (1646 ca.). In questa maniera ha inizio quel processo di interiorizzazione e di conoscenza di sé che il Nietzsche della Genealogia della morale chiama "cattiva coscienza", legata da una parte alla diffusione e penetrazione del cristianesimo nella cultura, e dall'altra al progressivo declino del linguaggio della tragedia, che perde il suo preponderante carattere musicale e la sua essenza sonora e mitica di fronte all'avanzare della consapevolezza retorica. ${ }^{43}$

Da questo punto di vista, è importante differenziare la posizione di Vico sia da quella di Nietzsche sia dal moralismo della tradizione cristiana. Infatti, a differenza di quello che pensava Nietzsche, la polemica di Vico, come quella che era stata condotta dallo stesso Salvator Rosa, riconosce che la menzogna non è un fatto costitutivo della poesia, e rimanda ad una realtà antropologica originaria del mito e della metafora, investendo il gusto decorativo per la mitologia proprio del barocco. Inoltre, per Vico, a differenza di quanto pensavano i moralisti, la poesia non deve nascondere le origini ferine dell'umanità, anche nel mondo moderno, ma al contrario deve rivelarle, è una poesia che si deve nutrire della memoria delle origini, non del formalismo letterario ed estetico del mondo barocco: solo in questa maniera potrà vedersi riconosciuta una funzione di primo piano nell'ambito della comunità umana e continuare a svolgere quella funzione etica che le è propria. In questo senso la poesia che Vico ha in mente non è allegorica, poiché al contrario della poesia barocca studiata con grande interesse da Benjamin, è una poesia che punta alla presenza dell'oggetto senza concentrarsi sulle tecniche di elusione dei significati. Una poesia in cui l'ombra del tempo non cancella l'elemento naturale, che tuttavia non viene esorcizzato in una visione ideale di natura intesa classicamente come permanenza e immutabilità, ma rimanda alla molteplice e rinnovata gnoseologia delle grandi passioni umane. ${ }^{44}$ L'approccio vichiano alle origini "ferine" della poesia e della civiltà umana consente al filosofo napoletano di evitare la separazione tra realtà e apparenza caratteristica della filosofia occidentale platonica e cristiana, impegnata nella difesa di valori spirituali di cui viene smarrita l'origine "umana troppo umana".

In questa prospettiva Vico non è disposto a riconoscere le Muse come 
ingannatrici, ciò a cui sembra alludere un luogo di Esiodo, prontamente ripreso dal Nietzsche, che ha sostenuto la necessità della teoria dell'artista "ingannatore". Infatti, nei discussi versi 27-28 della Teogonia le Muse affermano: "noi sappiamo dire molte menzogne simili alla verità; ma sappiamo anche, quando vogliamo, proclamare la verità". In genere si tende oggi ad ammettere che l'espressione "menzogne simili alla verità" vada riferita alla poesia omerica, condannata da Esiodo come menzogna, in nome di una nuova concezione della poesia che ne valorizzava il valore didascalico. L'osservazione del Nietzsche non tiene conto poi del fatto che per Esiodo anche l'aspetto ingannatore delle muse è divino e che il poeta nella Grecia arcaica è "maestro di verità". La verità del poeta ispirato è alquanto differente dalla concezione tradizionale della verità come opposizione vero/falso: è una verità intesa come alétheia, che non si oppone alla menzogna, e non può essere dimostrata, ma neanche contestata. Infatti, la sola opposizione significativa per il poeta della Grecia arcaica è quella di alétheia e di Lethé. ${ }^{46}$ Questo fatto non sfuggiva, invece, al Vico che ha trasformato la formula $A$ Iove principium Musae in criterio ermeneutico, e ha sottolineato il legame tra le Muse e Mnemosine.

Il tema della maschera che nel mondo antico ha forti radici con il culto dei morti e, al tempo stesso, sta alle origini della tragedia, per Vico rivelava uno stretto rapporto con il mito, e come il mito era vera narratio. Quando la sacralità del rito e del culto si esaurirono nacque la maschera in senso moderno. Nel contesto mitico e sacro era chiaro che la finzione della maschera non solo nascondeva il volto, ma lo rivelava anche, presentandolo nella sua verità. Anzi, la primitiva funzione della maschera nel culto dei morti non è quella di occultare, o di dissimulare, ma essa è lo strumento di una trasformazione unificatrice, in quanto da una parte essa elimina i limiti che dividono il mondo dei vivi da quello dei morti, e dall' altra comporta un'identificazione con il volto del morto e libera quanto di nascosto o dimenticato c'era in lui. La maschera diventa l'unico volto del morto. Allo stesso modo la maschera fu introdotta nella tragedia greca non per nascondere il personaggio, ma per permettergli di liberare energie e di esprimere una verità che il nudo volto non poteva esprimere. Nell'esperienza tragica della maschera si diventa satiro facendosi satiro per gli altri: chi partecipa alla mascherata rituale non ha altro volto che la propria maschera. ${ }^{47} \mathrm{~L}$ 'esperienza greca della maschera era dunque un'esperienza visiva, l'efficacia della ma- 
schera si realizza attraverso la vista. Gli occhi degli altri sono essenziali nel riconoscimento dei compagni di mascherata. L'esteriorità e non la conoscen$\mathrm{za}$ interiore sono all'origine del processo di identificazione personale. $\mathrm{E}$ negli occhi o sul volto degli altri, travestiti da satiri, che si riconosce, come in uno specchio, la propria identità di satiro. Lo sguardo dell'altro diventa come lo specchio in cui si può riconoscere la propria immagine. La finzione della maschera, la trasformazione che essa permetteva rivelava una verità profonda, come avveniva in ogni racconto metaforico, spontaneo, in ogni mito autentico. Per i Greci non c'è differenziazione linguistica tra maschera e viso e questo risulta particolarmente evidente anche nella pittura sepolcrale che si diffonde in Egitto e nel mondo ellenistico, dove la coscienza religiosa non distingueva la pittura o la maschera dal volto e non gli si contrapponeva: la maschera si pensava in rapporto al volto e prendeva senso e valore solo in rapporto ad esso. Infatti, come si è detto, la maschera nel culto dei morti non era una riduzione del defunto, ma una rivelazione della sua essenza spirituale più profonda che rompeva $\mathrm{i}$ limiti divisori tra la vita e la morte. ${ }^{48}$

La maschera che ci viene presentata nel quadro di Salvator Rosa allude invece all'artificio e alla possibilità della menzogna e dell'inganno che si offre come drammatica possibilità di un'esistenza priva di contenuto nella prosa della vita. Ne La menzogna gli occhi dell'altro, la figura sul lato destro della tela che fissa la figura in primo piano e la maschera che essa regge diventano non più lo specchio dell'identità tragica del personaggio, ma la testimonianza del suo sdoppiamento in maschera e volto. Per questo l'urna che campeggia sul lato sinistro del quadro sembra alludere al sepolcro, e all'interruzione della stessa continuità tra vita e morte che era all'origine dell'identificazione tra maschera e volto che si realizzava nella concezione religiosa primitiva e nelle antiche pitture sepolcrali. D'altro canto nella cultura barocca la morte cessa di essere una presenza, una realtà sostanziale e diventa simulacro. Simulacri della morte nel barocco non sono più solo le tombe, ma anche le istituzioni e l'intera storia umana che essendo svincolata da qualunque presupposto metafisico viene percepita come vuoto e come nulla. Il corpo stesso dell'uomo, come ha scritto Mario Perniola, è in fondo un'immagine, un travestimento, una maschera della morte..$^{49}$ Il quadro di Salvator Rosa, dunque, oltre a segnare il distacco dalle pulsioni originarie della vita, finisce per indicare anche l'allontanamento dalla visione sostan- 
ziale della morte, che viene sostituita da una sorta di simulazione della morte che non pone fine alla vita, ma al contrario appare la dimensione propria di una vita non più pensata in modo metáfisico.

Questo fenomeno si realizzava anche a proposito delle "maschere" delle divinità nella poesia contemporanea al Vico: esse, scriveva il filosofo e poeta napoletano, non rimandavano più alla vera narratio del mito, alla dimensione originaria della parola metaforica, ma all'artificio letterario e all'emergenza da una parte di una verità di tipo filosofico che non poteva più trovare espressione nella finzione del racconto mitico; e dall'altra di una "verità" legata a un segno che non ha più un legame diretto con la vita $\mathrm{e}$ si traduce necessariamente in menzogna, nel momento in cui rifiuta ogni determinazione linguistica legata al contenuto originario dell'esistenza. ${ }^{51}$ Ecco allora, che nella canzone di Vico, Origine, progresso e caduta della poesia, le muse, che un tempo per "felice natura" erano portate a cantare solo "virtù divine e grandi", sono ora costrette a cambiare la loro attitudine e a occuparsi della vita civile e di "privati ufizi", vivendo ormai solo nella finzione della "legge teatrale". È noto che Vico condannava la Commedia dell'Arte e in generale il ricorso all'ironia e al comico nel teatro. Si può parlare in un certo senso di un antisecentismo di Vico sulla linea di quello espresso da G. Vincenzo Gravina nella sua Ragione poetica $(1708) .{ }^{52} \mathrm{Ma}$ la considerazione delle origini ferine della poesia e dell'umanità dovevano spingere Vico ben al di là di una considerazione morale della letteratura come accadeva invece al Gravina.

La Commedia dell'Arte appartiene al genere dei detti arguti legati all'apparire improvviso in scena di personaggi ridicoli come le maschere di Pulcinella o di Arlecchino. Il favore di Vico appare invece rivolto alle "fabulae recte moratae", alle favole drammatiche in cui i personaggi non sono ridotti agli schemi della maschera, ma mantengono i loro caratteri individuali. Il diletto procurato da queste fabulae per Vico è degno del saggio, mentre la Commedia dell' Arte serve di passatempo agli uomini di mente debole o sfrenata, presso i quali il riso appare la conseguenza dell'insania e della dissoluzione dei vincoli razionali. ${ }^{\text {S3 }}$ Vico ritiene che l'ironia sia "formata dal falso in forza di una riflessione che prende maschera di verità". L'ironia non appartiene alla poesia vera dei primi uomini, così come il comico, e per Vico non coincide con il sublime, come si comincerà a sostenere nella stagione romantica che avvicinerà i due termini nella nozione di umorismo. ${ }^{54}$ 
Nella visione vichiana il comico e l'umorismo non trovano nessuna sublimazione e si presentano come un aspetto degradato dell'esistenza umana che non trova riscatto. La secolarizzazione e l'involgarimento delle muse fanno sì che non si cantino più le gesta di Achille, o dei grandi eroi: tutto quello che gli uomini e i poeti riescono a cantare e a celebrare sono ormai i giochi del circo, la finzione del "lottatore vincitore del gioco". 55 La poesia nel mondo degli uomini cessa perfino di cantare la bellezza divina e si volge a tributare "a bellezza mortale onori divini". ${ }^{56}$ Infatti, in questo generale processo di de-sublimazione della poesia e dell'esistenza umana, le lodi che un tempo venivano attribuite alle fanciulle divine vengono ora cantate per le donne. Al contrario le muse di cui ci parla Vico, le muse della Scienza nuova, sono e rimangono muse inquietanti, per riprendere il titolo di un famoso quadro di De Chirico che appare in qualche modo ispirato alle idee del filosofo napoletano. Nel quadro di De Chirico le muse hanno teste di manichini e sono prive di volto: non hanno occhi né bocca e sembrano alludere ad una sorta di maschera neutra.

Sembrano "feti di una umanità in gestazione", mentre la loro cecità rimanda ad una caratteristica dei poeti suggerita da Vico: "Omero stesso narra ciechi i poeti". D'altro canto, aggiunge Vico, "è proprietà di natura umana ch'i ciechi vagliono meravigliosamente nella memoria". ${ }^{57}$ La memoria per Vico è "madre delle muse". Il recupero delle origini primitive dell'umanità che nella Scienza nuova doveva indicare all'umanità la strada per un recupero di un'identità autentica, nella "metafisica schiarita" di De Chirico sembra dunque promuovere una perdita delle caratteristiche individuali, anche se i manichini di De Chirico non rappresentano una sorta di disumanizzazione, al contrario in essi è da vedere la ripresa di un'umanità arcaica, veggente ed eroica. È appena il caso di aggiungere in conclusione che i manichini di Giorgio De Chirico vivono in un'impotente non-identità, in una crisi di identità che si caratterizza come identità regredita piena di mistero e di enigma, cui sembra alludere anche un altro suo famoso quadro che si chiama Maschere e ci rimanda all'atmosfera perturbante che abbiamo incontrato nella Menzogna di Salvator Rosa. ${ }^{58}$

Vico considerava da un punto di vista antropologico i problemi di identità che stavano al centro della cultura barocca, e come Salvator Rosa pensava che solo ricorrendo alla memoria delle origini e delle passioni sublimi dei primi uomini fosse possibile dare vita ad una poesia moderna in 
grado di esprimere parole di verità riguardo alla condizione umana. In questo sia Vico che Salvator Rosa erano molto lontani dall'apologia poetica e retorica della menzogna e della maschera che si sviluppa nella cultura barocca. Lo stile barocco, come ha mostrato Heinrich Wölfflin, non può essere visto in termini di corpo umano, e non percepisce il senso della forma individuale. ${ }^{59}$ Il barocco raggiunge una nozione di sublime fondata su maschere, strumenti retorici, effetti di luce ed ombra e grandi volumi. Vico e Salvator Rosa comprendono questi sviluppi dell'arte e della cultura moderna, ma mantengono un'idea di sublime in cui l'essere umano, le passioni e il corpo sono ancora elementi significativi, non come espressione di valori estetici, ma come veicolo di un senso che non si vuole far consistere esclusivamente nel sistema dei segni.

MASSIMO LOLLINI

University of Oregon

${ }^{1}$ Cfr. G. Baudelaire, "Dell'essenza del riso", in Scritti sull'arte a cura di E. Raimondi e G. Guglielmi. Torino: Einaudi, 1981, 141.

${ }^{2}$ Cfr. A. Perrucci. Dell'Arte Rappresentativa premeditata e all'improvviso (1699), a cura di G. Bragaglia. Firenze: Sansoni, 1961. Cfr. anche F. Taviani. Commedia dell'arte e società barocca. Roma: Bulzoni, 1969 e Roberto Tessari. La Commedia dell'Arte nel Seicento: "industria" e "arte giocosa" della civiltà barocca. Firenze: Olschki, 1969.

${ }^{3}$ Cfr. A. Battistini e E. Raimondi, "Retoriche e poetiche dominanti", in Letteratura Italiana. Torino: Einaudi, 1984, vol. 3: Le forme del testo I. Teoria e poesia, 119 seg.

${ }^{4} \mathrm{Cfr}$. su questo punto Daniele Castellari, "Il teatro, l'immagine, il riso", in Intersezioni 9.1 (aprile 1989), 78.

${ }^{5} \mathrm{Cfr}$. J. Jallat, "Le masque ou l'art du déplacement. D'après un article de Jean Starobinski”, in Poètique, 2.8 (1971), 482.

${ }^{6}$ Sul tema della Unheimliche si può vedere il famoso saggio di Freud "Il perturbante", in Opere. Torino: Boringhieri, 1977, vol. 9, 81-118. Su questi aspetti cfr. anche Alessandro Fontana, "La scena", in Storia d'Italia. Torino: Einaudi, 1972, 790-866.

${ }^{7}$ Cfr. F. Nicolini. Vita di Arlecchino. Milano-Napoli: Ricciardi, 1958. L'aspetto spaventoso della maschera arcaica di Arlecchino (la barba ispida, il naso schiacciato e la protuberanza frontale residuo di un paio originario di corna) fanno concludere al Nicolini: "questa è maschera di dimonio". 16 . 204, ma vedi l'intero capitolo "Maschera" (201-18). Vedi anche A.G. Bragaglia. Pulcinella. Firenze: Sansoni, 1953, 
cfr. soprattutto il capitolo "La maschera e il naso" (63-76).

${ }^{8}$ Cfr. P. Toschi. Le origini del teatro italiano. Torino: Boringhieri, 1976. Vedi soprattutto il capitolo "Maschere demoniache nel Carnevale e nella commedia" (166-227).

${ }^{9} \mathrm{Ib} .167$ e 170.

${ }^{10}$ Sulle metamorfosi della maschera di Pulcinella si veda D. Scarfoglio e Luigi M. Lombardi Satriani. Pulcinella il mito e la storia. Milano: Leonardo, 1992.

${ }^{11} \mathrm{Cfr}$. Roberto Tessari, "Appunti per una definizione della maschera barocca”, in AA.VV. Da Dante al Novecento. Studi in onore di G. Getto, Milano, 1970, 274; e Daniele Castellari, "La retorica dell'improvviso", in Intersezioni, 6.3 (dicembre 1986), 437.

${ }^{12}$ G.B. Vico, "Origine, progresso e caduta della poesia", in Opere, a cura di Andrea Battistini. Milano: Mondadori, 1990, 275-80.

${ }^{13} \mathrm{Cfr}$. SNS, par. 533. Per quanto riguarda Ovidio si veda Metamorfosi 1.476. Clio per Vico è la seconda musa. Nella SNS l'attenzione di Vico si concentra esclusivamente sulla musa Urania. Il mito di Dafne viene illustrato anche nelle Dissertationes dove si sostiene che il principale merito di Apollo è quello di impedire l'accoppiamento casuale e dare leggi ai mariti. Cfr. "Dissertationes", in Opere Giuridiche. Il Diritto Universale. Introduzione di N. Badaloni. A cura di P. Cristofolini. Firenze: Sansoni, 1974, 907.

${ }^{14} \mathrm{Cfr}$. SNS, par. 910 . Vico ribadisce questa etimologia di personari come "vestir pelli di fiere" in $S N S$, par. 1033-34. La proposta di questa etimologia viene da G.C. Scaligero, Poetices libri septem 1.13.

${ }^{15}$ Op. cit., 209.

${ }^{16}$ Cfr. C. Ginzburg, "Mascherarsi da animali", in Storia nottuma. Una decifrazione dei sabba. Torino: Einaudi, 1989, 161-64.

${ }^{17} \mathrm{Cfr}$. SNS, 423.

${ }^{18}$ Cfr. W.F. Otto. Dioniso. Genova: il melangolo, 1990; vedi soprattutto il cap. "Il simbolo della maschera", $i b .$, 92-97.

${ }^{19} \mathrm{Si}$ veda a questo proposito B. Croce, "Di una lettera del Vico, che si credeva perduta, intorno alle maschere degli antichi", in La Critica, vol. 36 (1938), 389-91. Cfr. inoltre K. Kerenyi, "Uomo e maschera", in Miti e misteri. Torino: Boringhieri, 1990, 441-65. Per una introduzione generale al tema della maschera cfr. G. Allard, P. Lefort. Le masque. Paris: PUF, 1984. Per un'interpretazione del Vico in chiave antropologica si veda A. Battistini e E. Raimondi. "La visione antropologica della sapienza retorica: Vico", in "Retoriche e poetiche dominanti", cit., 138-44.

${ }^{20} \mathrm{Cfr}$. M. Foucault. Les mots et les choses. Une archèologie des sciences humaines. Paris: Gallimard, 1966, $318 \mathrm{sg}$.

${ }^{21}$ Su questo punto vedi R. Amheim. Il potere del centro. Torino: Einaudi, 1984, 227-228.

${ }^{22}$ Dalla voce "Maschere", in Pio Rossi, Convito morale per gli etici, economici 
e politici, portata seconda. Venezia: 1657; questo testo è in parte riprodotto in AA. VV. Elogio della menzogna, a cura di Salvatore S. Nigro (Palermo: Sellerio, 1990), con il titolo "Un vocabolario per la menzogna", la citazione si trova alla p. $144 .{ }^{23}$ Richard Alewyn. L'univers du baroque. Hambourg: Rowohlt Verlag, 1959, 51-53.

${ }^{24}$ Cfr. L. Salemo. Salvator Rosa. Milano: Edizioni per il Club del libro, 1963, 43.

${ }^{25} \mathrm{Cfr}$. Giovanni Baglione. Le vite de' pittori, scultori, architetti ed intagliatori, dal pontificato di Gregorio XIII del 1572 fino ai tempi di papa Urbano VIII nel 1642, scritte da Baglione Romano. Con la vita di Salvator Rosa Napoletano, pittore e poeta, scritta da Battista Passari, nuovamente aggiunta. Napoli, 1733.

${ }^{26} \mathrm{Cfr}$. P. Lacoue-Labarthe, "Le détour. Nietzsche et la rhétorique", in Poétique. Revue de theorie et d'analyse litteraires, 1971, n.5, 68.

${ }^{27}$ Cfr. R.A. Lanham. The Motives of Eloquence. Literary Rhetoric in the Renaissance. New Haven: Yale UP, 1976, 210-13.

${ }^{28} \mathrm{Cfr}$. H. Blumenberg. La leggibilità del mondo. Bologna: Il Mulino, 1984. Sul tema della maschera cfr. E. Gombrich, "La maschera e la faccia", in Id. L'immagine $e$ l'occhio. Torino: Einaudi, 1985. Il tema del volto come unicità e come testimonianza di un'esteriorità e molteplicità irriducibili ha trovato ampia trattazione in E. Levinas. Totalità e infinito. Milano: Jaca Book, 1990. Si veda soprattutto la sezione terza: "Il volto e l'esteriorità", 191-253.

${ }^{29}$ Op. cit., 94.

${ }^{30} \mathrm{Cfr}$. R. Otto. Das Heilige, Über das Irrationale in der Idee des Göttlichen und sein Verhältnis zum Rationalem. Stuttgart: Gotha 1923. Cfr. anche A. Fontana, cit., 849.

${ }^{31}$ Cfr. T. Accetto. Della Dissimulazione onesta. Edizione critica a cura di Salvatore S. Nigro. Presentazione di G. Manganelli. Genova: Costa \& Nolan, 1983; vedi cap. 2 e cap. 9. Sulla lotta politica nel Seicento si può vedere R. Villari. Elogio della dissimulazione. La lotta politica nel Seicento. Bari: Laterza, 1987. Cfr. inoltre T. Boccalini. Ragguagli di Parnaso e Pietra del Paragone Politico. A cura di G. Rua. Bari: Laterza, 1910; su Boccalini vedi anche A.A. Rosa. La Nuova scienza, il Barocco e la crisi della Controriforma. Roma-Bari: Laterza, 1974, 104.

${ }^{32}$ Cfr P. Rossi, cit., 127.

${ }^{33} \mathrm{Cfr}$. Adone, 14.36.4-6.

${ }^{34} \mathrm{Cfr}$. Della dissimulazione onesta, 1.26 .

${ }^{35} \mathrm{Cfr}$. Giuseppe Battista, "L'Apologia della menzogna", in AA.VV. Elogio della menzogna, cit., 63-81 (riprodotta da Id. Le giornate accademiche, Venezia, 1673). A proposito della nobilitazione del silenzio come regola di una vita saggia si veda la Descriptio silentii del ferrarese Celio Calcagnini (1479-1541) ib., 3 1-50.

${ }^{36} \mathrm{Cfr}$. Giorgio Santangelo. Il secentismo. Palumbo: 1958, 11-12.

${ }^{37}$ F. Nicolini. La giovinezza di Giambattista Vico (1668-1700). Bari: Laterza \& Figli, 1932, 150-51. 
${ }^{38}$ Cfr. Salvator Rosa. Il teatro della Politica. Sentenziosi afforismi della Prudenza. Edizione critica a cura di G. Baroni. Bologna: Collezione di Opere inedite o rare, 1991, dispensa 281. Cfr. gli aforismi 82, 276, 277, 280, 287 e 344.

${ }^{39} \mathrm{Ib}$. Cfr. aforisma 435.

${ }^{40}$ Agostino, De mendacio, cap. 4.

${ }^{41} \mathrm{Su}$ questo si veda Harald Weinrich, "Linguistica della menzogna", in Id. Metafora e menzogna: la serenità dell'arte. Bologna: il Mulino, 1976, 133-91. Alle considerazioni linguistiche di Weinrich si potrebbero affiancare quelle che vengono dalla filosofia del linguaggio (penso in particolare a J.L. Austin) e che vedono nella menzogna una proprietà implicita nel livello performativo del discorso. Cfr. su questo S. Felman. The Literary Speech Act. Don Juan with J.L. Austin, or Seduction in Two Languages. Ithaca (NY): Comell UP, 1983.

${ }^{42} \mathrm{Cfr}$. A. Cioranescu. Le Masque et le visage. Du baroque espagnol au classicisme francais. Genève: Droz S.A., 1983.

${ }^{43}$ F. Nietzsche. Genealogia della morale. Scelta di frammenti postumi (1886-1887). Milano: Mondadori, 1983, $66 \mathrm{sg}$. Su questo aspetto si veda P. Lacoue-Labarthe, cit., 67.

${ }^{44}$ Su Benjamin e il barocco si pud vedere G. Guglielmi, "Barocchi e moderni", in il Verri (marzo-giugno 1987), 97-110. Le analisi di Benjamin sul barocco sono contenute soprattutto in W. Benjamin. Il dramma barocco tedesco. Torino: Einaudi, 1971. Su Vico e le passioni si può vedere A. Battistini, "Vico and the Passions", in Teorie delle Passioni. A cura di E. Pulcini. Dordrecht, Boston, London: Kluwer Academic Publisher, 1989, 113-28.

${ }^{45} \mathrm{Cfr}$. F. Nietzsche. "Le Muse come ingannatrici", in Umano, troppo umano. Milano: Adelphi, 1981, vol. 2, 76.

${ }^{46} \mathrm{Cfr}$. M. Detienne, Les maîtres de vérité dans la Grèce archaïque, cit., 27. Su problematica relativa alle muse nella poesia di Esiodo si veda Poetica Pre-Platonica. Testimonianze e Frammenti, testo, traduzione e commento a cura di Giuliana Lanata. Firenze: La Nuova Italia, 1963, 20 seg.; S. Accame, "L'invocazione alla Musa e la "Verita' in Omero e in Esiodo", in Rivista di filologia e di istruzione classica, 1963, 35, 257-81 e 385-415; P. Pucci, "The True and False Discourse in Hesiod", in Id. Hesiod and the Language of Poetry. Baltimore and London: The Johns Hopkins UP, $1977,8-44$.

${ }^{47}$ Cfr. K. Kerenyi, "Uomo e maschera", cit., 444-45; e F. Frontisi-Ducroux, "Senza maschera né specchio: l'uomo greco e i suoi doppi", in AA.VV. La maschera, il doppio e il ritratto. A cura di M. Bettini. Roma-Bari: Laterza, 1991, 130-58.

${ }^{48} \mathrm{Cfr}$. P. Florenskij. Le porte regali. Saggio sull'icona. Milano: Adelphi, 1977, 190 sg.; e F. Frontisi-Ducroux, cit.

${ }^{49} \mathrm{Cfr}$. M. Perniola. La società dei simulacri. Bologna: Cappelli, 1980, 96.

so Ibidem. Pemiola collega l'idea della modema simulazione della morte alla tradizione gesuitico-barocca. In particolare gli Esercizi spirituali di Ignazio 
consigliano l'elezione e la simulazione della morte allo scopo di maturare un atteggiamento di indifferenza e di umiltà verso il mondo. La simulazione della morte rimuove la volontà del soggetto trasformandola in pulsione inconscia svincolata da qualunque contenuto determinato. In questo modo la proposta ignaziana finirebbe per incontrarsi con la teatralizzazione della vita caratteristica della società barocca.

${ }^{51}$ Il contrasto tra la verità espressa nel sapere tragico del mito e quella che trova espressione nel sapere filosofico risale alla filosofia platonica e alla Grecia del V sec. d.C. Qui ha origine una vera e propria "battaglia della verità" che continua con il Vico. Su questo punto cfr. F. Rella. La battaglia della verità. Milano: Feltrinelli, 1986.

${ }^{52}$ Sull' antisecentismo del Gravina si può vedere G. Santangelo, cit., 35-36.

${ }^{53}$ Cfr. G.B. Vico, "Vindiciae", ora in Opere a cura di F. Nicolini (Napoli: Ricciardi, 1953), con il titolo "Dell'ingegno umano dei detti acuti e arguti e del riso", 926-32.

${ }^{54} \mathrm{Cfr}$. SNS, 408. Sulla problematica del rapporto tra sublime e comico nell'estetica del Settecento si veda G. Costa, "Il comico e il sublime nella cultura italiana del primo Settecento", in Intersezioni, 1.3 (1981), 555-73. Costa fa notare che l'interesse storicistico-sociologico di Vico gli fa cogliere un legame dialettico tra sublime e comico, ma poi, come era accaduto anche al Gravina il sublime esercita una "funzione inibitoria" nei confronti del comico. Cfr. $i b ., 573$.

${ }^{55} \mathrm{Cfr}$. G.B. Vico, "Origine, progresso e caduta della poesia", cit., 311.

${ }^{56}$ Ibidem.

${ }^{57} \mathrm{Cfr}$. SNS, par. 870 e 871.

${ }^{58}$ Sull'influenza di Vico su De Chirico e Savinio cfr. Maurizio Calvesi. La metafisica schiarita. Da De Chirico a Carrà, da Morandi a Savinio. Milano: Feltrinelli, 1982, 89-94.

${ }^{59} \mathrm{Cfr}$. H. Wölfflin. Rinascimento e Barocco. Con un saggio introduttivo di Simone Viani. Firenze: Vallecchi, 1988. 